Research Paper

\title{
Use of CEA and CA15-3 to Predict Axillary Lymph Node Metastasis in Patients with Breast Cancer
}

\author{
San-Gang $\mathrm{Wu}^{1^{*}}$, Zhen-Yu He ${ }^{2 *}$, Hong-Yue Ren ${ }^{3^{*}}$, Li-Chao Yang ${ }^{4}$, Jia-Yuan Sun², Feng-Yan Li², Ling Guo ${ }^{5 凶}$, \\ Huan-Xin $\operatorname{Lin}^{2}$ \\ 1. Xiamen Cancer Center, Department of Radiation Oncology, the First Affiliated Hospital of Xiamen University, Xiamen, People's Republic of China; \\ 2. Sun Yat-sen University Cancer Center, State Key Laboratory of Oncology in South China, Department of Radiation Oncology, Collaborative Innovation \\ Center of Cancer Medicine, Guangzhou, People's Republic of China; \\ 3. Biobank, The First Affiliated Hospital of Xiamen University, Xiamen, People's Republic of China; \\ 4. Department of Basic Medical Science, Medical College, Xiamen University, Xiamen, People's Republic of China; \\ 5. Sun Yat-sen University Cancer Center, State Key Laboratory of Oncology in South China, Department of Nasopharyngeal Carcinoma, Collaborative Inno- \\ vation Center of Cancer Medicine, Guangzhou, People's Republic of China. \\ * Equally contributors \\ $\square$ Corresponding authors: Ling Guo, Sun Yat-sen University Cancer Center, State Key Laboratory of Oncology in South China, Department of Nasopharyngeal \\ Carcinoma, Collaborative Innovation Center of Cancer Medicine, Guangzhou, People's Republic of China. Tel. +86 2087343543 Fax. +86 2087343392 E-mail. \\ linhx@sysucc.org.cn or Huan-Xin Lin, Sun Yat-sen University Cancer Center, State Key Laboratory of Oncology in South China, Department of Radiation On- \\ cology, Collaborative Innovation Center of Cancer Medicine, Guangzhou, People's Republic of China. Tel. +86 2087343543 Fax. +86 2087343392 E-mail. \\ guol@sysucc.org.cn.
}

(c) Ivyspring International Publisher. Reproduction is permitted for personal, noncommercial use, provided that the article is in whole, unmodified, and properly cited. See http://ivyspring.com/terms for terms and conditions.

Received: 2015.06.30; Accepted: 2015.09.19; Published: 2016.01.01

\begin{abstract}
Purpose: The clinical significance of preoperative serum levels of carcinoembryonic antigen (CEA) and cancer antigen 15-3 (CA15-3) in breast cancer is controversial. The purpose of this study was to assess the clinical value of preoperative serum levels of CEA and CA 15-3 on the risk of axillary lymph node metastasis (ALNM) in patients with breast cancer.

Methods: This retrospective study analyzed 1148 breast cancer patients whose preoperative CEA and CA 15-3 levels were measured. The association of these tumor markers and clinicopathologic parameters with ALNM was determined by univariate and multivariate analysis.

Results: A median of 15 lymph nodes were removed. Seven hundred seventy-eight (67.8\%) patients had node-negative disease and $370(32.2 \%)$ had ALNM. Univariate analysis showed that tumor location $(P=0.024)$, stage $(P=0.001)$, grade $(P<0.001)$, lymphovascular invasion $(\mathrm{LVI})(P<$ $0.001)$, CEA level $(P<0.001)$, CA15-3 level $(P<0.001)$, and breast cancer subtype (BCS) $(P<$ 0.001 ) were significantly associated with ALNM. ALNM was present in $4.5 \%$ of patients with normal CEA and $11.6 \%$ of patients with elevated CEA. ALNM was present in $8.0 \%$ of patients with normal CA15-3 and $17.0 \%$ of patients with high CA15-3. Multivariate logistic regression analysis showed that tumor location, stage, grade, LVI, CEA, CA15-3, and BCS were significantly and independently associated with ALNM $(P<0.05$ for all).

Conclusion: The probability of ALNM was greater in patients with elevated preoperative serum levels of CEA and CA15-3. CEA and CA15-3 appear to be independent predictors of ALNM in breast cancer.
\end{abstract}

Key words: Breast cancer, axillary lymph node metastases, CEA, CA15-3.

\section{Introduction}

Axillary lymph node dissection (ALND) is an important procedure in the treatment of breast cancer because the results are used to predict patient prog- nosis and guide adjuvant therapy. However, complications of ALND can adversely affect patient quality-of-life (QOL) (1-3). An increasing number of pa- 
tients experience these adverse effects because improvements in adjuvant therapy have improved survival rates. Therefore, it is very important to determine the effect of surgical procedures on the QOL of patients with breast cancer.

Numerous recent breast cancer studies have focused on the clinical significance of sentinel lymph node biopsy (SLNB). The results of the Z0011 trial showed that ALND did not affect the survival rate of patients with 0-3 positive sentinel nodes, and that sentinel lymph node biopsy can significantly improve patient QOL $(4,5)$. However, there is still controversy regarding the need for ALNB in patients with breast cancer. The $13^{\text {th }}$ St. Gallen International Breast Cancer Conference (2013) only recommended SLNB for some patients in order to avoid ALND (6). Preoperative identification of axillary lymph node status is an important basis for selection of the most appropriate surgical procedure for the treatment of lymph nodes. However, there are currently no established standards. Therefore, a reliable and simple method is needed to predict the axillary lymph node status of patients with breast cancer.

Previous studies reported that 2 common markers of breast cancer, carcinoembryonic antigen (CEA) and cancer antigen 15-3 (CA15-3), can predict patient prognosis (7-10). However, there have been no studies of the value of CEA and CA15-3 in predicting axillary lymph node status in patients with breast cancer. In the present study, we retrospectively analyzed the clinicopathologic factors and tumor markers of patients with breast cancer to determine whether CEA and CA15-3 can predict axillary lymph node status of these patients.

\section{Patients and methods}

\section{Patients}

The records of female patients with breast cancer who were admitted to the Sun Yat-Sen University Cancer Center (Guangzhou) between March 2001 and January 2007 were retrospectively reviewed. Each included patient had unilateral invasive breast cancer without distant metastasis at diagnosis, mastectomy or breast-conserving surgery and ALND, measurement of CEA and CA15-3 before surgery, complete surgical resection of the tumor and no residual tumor based on pathological exam, no neoadjuvant therapy before surgery, complete results of tests for the estrogen receptor (ER), progesterone receptor (PR), and human epidermal growth factor receptor 2 (Her 2), and no sign of malignant tumors in other organs at diagnosis.

\section{Methods for measurement of CEA, CA15-3, and other pathological factors}

Five $\mathrm{mL}$ of venous blood was collected from each patient upon admission. Serum was obtained after centrifugation, and was placed in a refrigerated tube and immediately preserved at $-80^{\circ} \mathrm{C}$.

Serum levels of CEA and CA15-3 were determined with the Roche E170 modular analytics immunoassay analyzer. We considered the normal range of CEA as $0-5 \mathrm{ng} / \mathrm{mL}$ and the normal range of CA15-3 as $0-25 \mathrm{U} / \mathrm{mL}$. ER- and PR-positivity was defined by the presence of at least $1 \%$ positive cells based on immunohistochemical results. Her-2 positivity was defined as immunohistochemical grade of $3+$ or $2+$, as determined by fluorescence in situ hybridization (FISH). For measurement of Ki-67, the ratio of positive cells to total cells in a selected microscopic field was determined; "negative" was defined as less than $10 \%$ positivity, "weakly positive" as $10-25 \%$ positivity, "positive" as $26-50 \%$ positivity, and "strongly positive" as more than $50 \%$ positivity. Based on our previous studies, $25 \%$ positivity was used as the cut-off point (11).

\section{Factors associated with axillary lymph node status}

The axillary lymph node status was predicted using clinicopathologic and immunohistochemical factors including age, menopausal status, tumor location, tumor stage, grade, lymphovascular invasion (LVI), and breast cancer subtype (BCS). Tumor location was classified as outer (upper lateral and lower lateral) quadrant, inner (upper medial and lower medial) quadrant, and central. All cancers were categorized according to subtype: (i) ER+ and/or PR+, Her2+ (HR+/HER2-); (ii) ER+ and/or PR+, Her2+ (HR+/HER2+); (iii) ER-, PR-, and Her2+ (HR-/HER2+); or (iv) ER- and PR- and Her2(HR-/HER2-).

\section{Statistical analysis}

All data were analyzed using the SPSS statistical software package (version 16.0; IBM, Armonk, NY, USA). The relationship between clinicopathologic factors, tumor markers, and ALNM was examined by univariate analysis using the $\chi^{2}$ test and Fisher's exact test. Then multivariate logistic regression analysis was used, in which factors that were statistically significant in the univariate analysis were entered into multivariate logistic regression analysis. A $P$-value less than 0.05 was considered significant. 


\section{Results}

\section{Clinicopathologic status and lymph node re- section}

A total of 1148 patients were retrospectively enrolled from March 2001 to January 2007 (Table 1). One thousand and seventy-six patients (93.7\%) received mastectomy and 72 patients $(6.3 \%)$ received breast conserving surgery. The median age was 47 years (range: $21-90$ years). Serum CEA was elevated in 78 cases $(6.8 \%)$ and serum CA15-3 was elevated in 125 cases $(10.9 \%)$. Analysis of BCS indicated that $53.6 \%$ of patients had HR+/HER2- subtype, $16.2 \%$ had HR+/HER2+ subtype, $15.2 \%$ had HR-/HER2+ tumors, and $15.0 \%$ had HR-/HER2- (triple negative, TN) tumors. All patients underwent ALND. The median number of removed lymph nodes was 15 (range: 5-45). Overall, 778 patients $(67.8 \%)$ had axillary node-negative disease and 370 patients $(32.2 \%)$ had ALNM.

Table 1. Clinicopathologic characteristics of patients with breast cancer who presented with and without axillary lymph node metastases

\begin{tabular}{|c|c|c|c|c|}
\hline Characteristics & $\mathrm{N}(\%)$ & $\begin{array}{l}\text { Lymph node } \\
\text { negative (\%) }\end{array}$ & $\begin{array}{l}\text { Lymph node } \\
\text { positive (\%) }\end{array}$ & $p$-value \\
\hline \multicolumn{5}{|l|}{ Age (year) } \\
\hline$\leq 35$ & $140(12.2)$ & $92(11.8)$ & $48(13.0)$ & \multirow[t]{2}{*}{0.579} \\
\hline$>35$ & $1008(87.8)$ & $686(88.2)$ & $322(87.0)$ & \\
\hline \multicolumn{5}{|l|}{ Menopausal status } \\
\hline Premenopausal & $736(64.1)$ & $496(63.8)$ & $240(64.9)$ & \multirow[t]{2}{*}{0.714} \\
\hline Postmenopausal & $412(35.9)$ & $282(36.2)$ & $130(35.1)$ & \\
\hline \multicolumn{5}{|l|}{ Tumor location* } \\
\hline Outer quadrant & $725(63.5)$ & 475 (61.3) & $250(68.1)$ & \multirow[t]{3}{*}{0.024} \\
\hline Inner quadrant & $278(24.3)$ & $207(26.7)$ & $71(19.3)$ & \\
\hline Central & 139 (12.2) & $93(12.0)$ & $46(12.5)$ & \\
\hline \multicolumn{5}{|l|}{ Tumor stage } \\
\hline pT1 & $462(40.2)$ & $341(43.8)$ & $121(32.7)$ & \multirow[t]{4}{*}{0.001} \\
\hline pT2 & $588(51.2)$ & $394(50.6)$ & $194(52.4)$ & \\
\hline pT3 & $59(5.1)$ & $28(3.6)$ & $31(8.4)$ & \\
\hline pT4 & $39(3.4)$ & $15(2.0)$ & $24(6.5)$ & \\
\hline \multicolumn{5}{|l|}{ Grade* } \\
\hline I & 118 (10.6) & $102(13.1)$ & $16(4.8)$ & \multirow[t]{3}{*}{$<0.001$} \\
\hline II & $523(47.1)$ & 375 (48.2) & $148(44.4)$ & \\
\hline III & $470(42.3)$ & $301(38.7)$ & $169(50.8)$ & \\
\hline \multicolumn{5}{|l|}{$\begin{array}{l}\text { Lymphovascular } \\
\text { invasion }\end{array}$} \\
\hline Negative & 1097 (95.6) & $769(98.8)$ & $328(88.6)$ & \multirow[t]{2}{*}{$<0.001$} \\
\hline Positive & $51(4.4)$ & $9(1.2)$ & 42 (11.6) & \\
\hline \multicolumn{5}{|l|}{ CEA } \\
\hline Normal & $1070(93.2)$ & $743(95.5)$ & 327 (88.4) & \multirow[t]{2}{*}{$<0.001$} \\
\hline High & $78(6.8)$ & $35(4.5)$ & 43 (11.6) & \\
\hline \multicolumn{5}{|l|}{ CA15-3 } \\
\hline Normal & $1023(89.1)$ & $716(92.0)$ & $307(83.0)$ & \multirow[t]{2}{*}{$<0.001$} \\
\hline High & $125(10.9)$ & $62(8.0)$ & $63(17.0)$ & \\
\hline \multicolumn{5}{|l|}{ Ki $67(\%)^{*}$} \\
\hline$\leq 25$ & 447 (56.6) & $316(57.8)$ & 131 (53.9) & \multirow[t]{2}{*}{0.312} \\
\hline$>25$ & $343(43.4)$ & $231(42.2)$ & $112(46.1)$ & \\
\hline \multicolumn{5}{|c|}{ Breast cancer subtype } \\
\hline HR+/HER2- & 615 (53.6) & $422(54.2)$ & $193(52.2)$ & \multirow[t]{4}{*}{$<0.001$} \\
\hline HR+/HER2+ & $186(16.2)$ & 107 (13.8) & 79 (21.3) & \\
\hline HR-/HER2+ & 175 (15.2) & 114 (14.6) & $61(16.5)$ & \\
\hline HR-/HER2- & $172(15.0)$ & 135 (17.4) & $37(10.0)$ & \\
\hline
\end{tabular}

With missing data.

\section{Univariate analysis of risk factors with ALNM}

Table 1 shows the results of univariate analysis of the correlation between clinicopathologic factors and ALNM. Tumor location $(p=0.024)$, tumor stage $(p$ $=0.001)$, grade $(p<0.001)$, lymphovascular invasion (LVI) $(p<0.001)$, CEA $(P<0.001)$, CA15-3 $(p<0.001)$, and BCS $(p<0.001)$ were significantly associated with ALNM. Age, menopausal status, and Ki-67 were not associated with ALNM ( $p>0.05$ for all). A total of $4.5 \%$ of patients with normal CEA levels had ALNM, and $11.6 \%$ of those with high levels had ALNM. Similarly, a total of $8.0 \%$ of patients with normal CA15-3 levels had ALNM, and $17.0 \%$ of those with high levels had ALNM.

\section{Multivariate analysis of risk factors with ALNM}

Table 2 shows the results of multivariate logistic regression analysis of factors that were significant in the univariate analysis. Model 1 includes clinicopathologic factors, but not tumor markers. Model 2 includes the same clinicopathologic factors and tumor markers. In Model 1, tumor location, tumor stage, grade, LVI, and BCS were significant predictive factors for ALNM ( $p<0.05$ for all). In Model 2, all of these factors as well as CEA and CA15-3 levels were significantly and independently associated with ALNM ( $p<0.05$ for all). Patients with normal levels of CEA and CA15-3 had the lower risk for ALNM. Relative to patients with normal levels of CEA, those with high levels of CEA were significantly more likely to have ALNM (odds ratio [OR] $=2.139,95 \%$ confidence interval $[\mathrm{CI}]=1.261-3.630, p=0.005)$. Relative to patients with normal levels of CA15-3, those with high levels of CA15-3 were significantly more likely to have ALNM $(\mathrm{OR}=2.012,95 \% \mathrm{CI}=1.321-3.064, p=$ $0.001)$.

\section{Discussion}

In the present study, we investigated the value of serum levels of CEA and CA15-3 in predicting ALNM in patients with breast cancer. Our results showed that elevated preoperative serum levels of CEA and CA-153 were significantly and independently associated with ALNM.

Axillary lymph node status is an important factor that affects the prognosis of breast cancer. Except for obtaining the results of pathological examination after SLNB or ALND, there is no definite standard to predict axillary lymph node status. Previous studies of patients with breast cancer reported that the risk factors for ALNM included age, tumor stage, BCS, tumor grade, and LVI (12-16). In the present study, we also found that tumor location, tumor stage, tumor grade, LVI, and BCS were independent factors predicting ALNM. However, no previous studies have 
assessed the clinical value of CEA and CA15-3 in predicting ALNM in patients with breast cancer. Our previous studies indicated that serum levels of CEA and CA15-3 could be used to predict the survival of patients with breast cancer (7). The results of the present study suggest that CEA and CA15-3 can be used to predict ALNM as well as hematogenous metastasis of breast cancer.

Table 2. Multiple logistic regression analysis of the association of clinicopathologic characteristics with axillary lymph node metastases in patients with breast cancer.

\begin{tabular}{|c|c|c|c|}
\hline Characteristics & HR & $\mathrm{CI}$ & $p$-value \\
\hline \multicolumn{4}{|l|}{ Model 1} \\
\hline \multicolumn{4}{|l|}{ Tumor location } \\
\hline Inner quadrant vs. outer quadrant & 0.635 & $0.450-0.896$ & 0.010 \\
\hline Central vs. outer quadrant & 0.825 & $0.535-1.272$ & 0.383 \\
\hline \multicolumn{4}{|l|}{ Tumor stage } \\
\hline pT2 vs. pT1 & 1.290 & $0.961-1.732$ & 0.090 \\
\hline pT3 vs. pT1 & 2.586 & $1.405-4.762$ & 0.002 \\
\hline pT4 vs. pT1 & 3.779 & $1.834-7.788$ & $<0.001$ \\
\hline \multicolumn{4}{|l|}{ Grade } \\
\hline II vs. I & 2.266 & $1.258-4.085$ & 0.006 \\
\hline III vs. I & 3.456 & $1.925-6.206$ & $<0.001$ \\
\hline \multicolumn{4}{|l|}{ Lymphovascular invasion } \\
\hline Positive vs. negative & 9.434 & $4.430-20.093$ & $<0.001$ \\
\hline \multicolumn{4}{|l|}{ Breast cancer subtype } \\
\hline HR+/HER2- vs. HR-/HER2- & 1.258 & $0.819-1.931$ & 0.294 \\
\hline HR+/HER2+ vs. HR-/HER2- & 1.869 & $1.133-3.084$ & 0.014 \\
\hline HR-/HER2+ vs. HR-/HER2- & 1.640 & $0.986-2.729$ & 0.057 \\
\hline \multicolumn{4}{|l|}{ Model 2} \\
\hline \multicolumn{4}{|l|}{ Tumor location } \\
\hline Inner quadrant vs. outer quadrant & 0.626 & 0.443-0.885 & 0.008 \\
\hline Central vs. outer quadrant & 0.818 & $0.529-1.265$ & 0.366 \\
\hline \multicolumn{4}{|l|}{ Tumor stage } \\
\hline pT2 vs. pT1 & 1.321 & $0.984-1.775$ & 0.064 \\
\hline pT3 vs. pT1 & 2.530 & $1.365-4.691$ & 0.003 \\
\hline pT4 vs. pT1 & 3.143 & $1.494-6.612$ & 0.003 \\
\hline \multicolumn{4}{|l|}{ Grade } \\
\hline II vs. I & 2.192 & $1.225-3.922$ & 0.008 \\
\hline III vs. I & 3.482 & $1.949-6.220$ & $<0.001$ \\
\hline \multicolumn{4}{|l|}{ Lymphovascular invasion } \\
\hline Positive vs. negative & 10.061 & $4.679-21.632$ & $<0.001$ \\
\hline \multicolumn{4}{|l|}{ CEA } \\
\hline High vs. normal & 2.139 & $1.261-3.630$ & 0.005 \\
\hline \multicolumn{4}{|l|}{ CA15-3 } \\
\hline High vs. normal & 2.012 & $1.321-3.064$ & 0.001 \\
\hline \multicolumn{4}{|l|}{ Breast cancer subtype } \\
\hline HR+/HER2- vs. HR-/HER2- & 1.283 & $0.832-1.979$ & 0.259 \\
\hline HR+/HER2+ vs. HR-/HER2- & 1.790 & $1.077-2.974$ & 0.025 \\
\hline HR-/HER2+ vs. HR-/HER2- & 1.522 & $0.904-2.563$ & 0.114 \\
\hline
\end{tabular}

At present, the molecular mechanism underlying the association of CEA and CA15-3 with ALNM is unknown. Previous research reported that adhesion molecules play a key role in tumor invasion of the lymphatic lumen (17), and that CEA may act as a cell adhesion molecule during tumor invasion (18). CA15-3 is a member of the mucin-1 (MUC-1) family of glycoproteins. MUC-1 expression has tissue specificity, and is mainly expressed in the epithelial tissue of the normal and cancerous mammary glands, but not in the mesenchyme-derived lymph nodes $(19,20)$. This may explain the positive association of serum levels of CEA and CA15-3 with ALNM in our patients. In agreement, Kim et al. measured the concentrations of CA-15-3 and CEA in fine-needle aspirates of axillary lymph nodes and found that their concentrations could be useful for pre-surgical diagnosis of ALNM in patients with breast cancer (21).

Previous studies indicated that ALNM was less common in patients with TN breast cancer than in those with other BCS $(12,16)$. In the present study, we also found that ALNM was less common in patients with TN breast cancer than in those who were HR+/HER2+. Our previous studies also showed that CEA levels were lower in patients with $\mathrm{TN}$ breast cancer than in those with other subtypes $(p=0.002)$ (7). Taken together, these findings suggest that TN breast cancer has unique biological behaviors.

At present, there is controversy regarding the use of CEA and CA15-3 in the diagnosis of breast cancer. The European Society for Medical Oncology (ESMO) and the European Group on Tumor Markers (EGTM) suggested that routine measurement of tumor markers such as CEA and CA15-3 should be performed in patients with breast cancer $(22,23)$. However, the American Society of Clinical Oncology (ASCO) does not recommend routine measurement of CEA, CA15-3, or other tumor markers for patients with breast cancer (24). A report that used Surveillance, Epidemiology and End Results (SEER) records $(39,650$ patients) suggested that tumor markers (including CEA and CA153) should not be routinely measured in patients with early-stage breast cancer. However, this study only enrolled patients who were at least 65 years-old (25). Although there have not yet been randomized controlled trials, the results presented here and additional studies support the use of CEA and CA15-3 for prediction of the prognosis of breast cancer (7-10). More generally, routine measurement of tumor markers can be helpful for predicting the prognosis and the axillary lymph node status of breast cancer patients.

SLNB is appropriate for some early-stage breast cancers, and the results can predict axillary lymph node status and help to determine whether ALND is necessary. Most patients in the present study had total mastectomy with ALND, and both were outside the Z0011 selection criteria (i.e. patients who need ALND). However, based on our study, we believe that detailed evaluation of axillary lymph node status is necessary for CEA- and CA15-3-positive patients during SLNB before surgery.

The present study had some limitation. This was a single-center retrospective analysis, and this may 
have biased the results. Our findings may not be applicable to other populations. Second, SLNBs were not performed in our patients, so we do not know whether serum levels of CEA and CA15-3 can be used to predict the sentinel lymph node status in patients with breast cancer.

\section{Conclusion}

In conclusion, the results of the present study show that patients with breast cancer who test positive for CEA and CA15-3 before surgery have increased risk of ALNM. Therefore, clinicians should consider the use of preoperative serum levels of CEA and CA15-3 for guiding locoregional management decisions if further confirmed in larger sample size studies.

\section{Abbreviations}

ALND, axillary lymph node dissection; QOL, quality-of-life; SLNB, sentinel lymph node biopsy; CEA, carcinoembryonic antigen; CA15-3, cancer antigen 15-3; ER, estrogen receptor; $\mathrm{PR}$, progesterone receptor; Her 2, human epidermal growth factor receptor 2; FISH, fluorescence in situ hybridization; LVI, lymphovascular invasion; BSC, breast cancer subtype; $\mathrm{TN}$, triple negative; $\mathrm{OR}$, odds ratio; $\mathrm{CI}$, confidence interval; ASCO, American Society of Clinical Oncology; ESMO, European Society for Medical Oncology; SEER, Surveillance, Epidemiology and End Results.

\section{Acknowledgments}

This work was supported by grants from the National Natural Science Foundation of China (No. 81402527), the Sci-Tech Office of Guangdong Province (No. 2013B021800157, 2013B021800458), and the Youth Foundation of Fujian Provincial Health and Family Planning Commission (No. 2014-2-63).

\section{Conflict of interest}

No any actual or potential conflicts of interest exist.

\section{References}

1. Kuehn T, Klauss W, Darsow M, et al. Long-term morbidity following axillary dissection in breast cancer patients--clinical assessment, significance for life quality and the impact of demographic, oncologic and therapeutic factors. Breast Cancer Res Treat 2000;64(3):275-86.

2. Kwan W, Jackson J, Weir LM, et al. Chronic arm morbidity after curative breast cancer treatment: prevalence and impact on quality of life. J Clin Oncol 2002;20(20):4242-8

3. Erickson VS, Pearson ML, Ganz PA, et al. Arm edema in breast cancer patients. J Natl Cancer Inst 2001;93(2):96-111.

4. Giuliano AE, McCall L, Beitsch P, et al. Locoregional recurrence after sentinel lymph node dissection with or without axillary dissection in patients withsentinel lymph node metastases: the American College of Surgeons Oncology Group Z0011 randomized trial. Ann Surg 2010;252(3):426-32; discussion 432-3.

5. Giuliano $\mathrm{AE}$, Hunt $\mathrm{KK}$, Ballman $\mathrm{KV}$, et al Axillary dissection vs no axillary dissection in women with invasive breast cancer and sentinel node metastasis: arandomized clinical trial. JAMA 2011;305(6):569-75.
6. Goldhirsch A, Winer EP, Coates AS, et al. Personalizing the treatment of women with early breast cancer: highlights of the St Gallen International Expert Consensus on the Primary Therapy of Early Breast Cancer 2013. Ann Oncol 2013;24(9):2206-23

7. Wu SG, He ZY, Zhou J, et al. Serum levels of CEA and CA15-3 in different molecular subtypes and prognostic value in Chinese breast cancer. Breast 2014;23(1):88-93.

8. Lee JS, Park S, Park JM, et al. Elevated levels of preoperative CA 15-3 and CEA serum levels have independently poor prognostic significance in breast cancer. Ann Oncol 2013;24(5):1225-31.

9. Molina R, Auge JM, Farrus B, et al. Prospective evaluation of carcinoembryonic antigen (CEA) and carbohydrate antigen 15.3 (CA 15.3) in patients with primary locoregional breast cancer. Clin Chem 2010;56(7):1148-57.

10. Park BW, Oh JW, Kim JH, et al. Preoperative CA 15-3 and CEA serum levels as predictor for breast cancer outcomes. Ann Oncol 2008;19(4):675-81.

11. Li FY, Wu SG, Zhou J, et al. Prognostic value of Ki-67 in breast cancer patients with positive axillary lymph nodes: a retrospective cohort study. PLoS One 2014;9(2):e87264.

12. Ugras S, Stempel M, Patil S, et al. Estrogen receptor, progesterone receptor, and HER2 status predict lymphovascular invasion and lymph nodeinvolvement. Ann Surg Oncol 2014;21(12):3780-6.

13. Gangi A, Mirocha J, Leong T, et al. Triple-negative breast cancer is not associated with increased likelihood of nodal metastases. Ann Surg Oncol 2014;21(13):4098-103.

14. Viale G, Zurrida S, Maiorano E, et al. Predicting the status of axillary sentinel lymph nodes in 4351 patients with invasive breast carcinoma treated in asingle institution. Cancer. 2005;103(3):492-500.

15. Greer LT, Rosman M, Charles Mylander W, et al. A prediction model for the presence of axillary lymph node involvement in women with invasive breast cancer: afocus on older women. Breast J 2014;20(2):147-53

16. Crabb SJ, Cheang MC, Leung S, et al. Basal breast cancer molecular subtype predicts for lower incidence of axillary lymph node metastases in primarybreast cancer. Clin Breast Cancer 2008;8(3):249-56.

17. Nathanson SD. Insights into the mechanisms of lymph node metastasis. Cancer 2003:98(2):413-23.

18. Benchimol S, Fuks A, Jothy S, et al. Carcinoembryonic antigen, a human tumor marker, functions as an intercellular adhesion molecule. Cell 1989;57(2):327-34.

19. Mirabelli $\mathrm{P}$, Incoronato $\mathrm{M}$. Usefulness of traditional serum biomarkers for management of breast cancer patients. Biomed Res Int 2013;2013:685641.

20. Duffy MJ, Evoy D, McDermott EW. CA 15-3: uses and limitation as a biomarker for breast cancer. Clin Chim Acta 2010;411(23-24):1869-74.

21. Kim MJ, Park BW, Lim JB, et al. Axillary lymph node metastasis: CA-15-3 and carcinoembryonic antigen concentrations in fine-needle aspiratesfor preoperative diagnosis in patients with breast cancer. Radiology 2010;254(3):691-7.

22. Cardoso F, Harbeck N, Fallowfield L et al. Locally recurrent or metastatic breast cancer: ESMO Clinical Practice Guidelines for diagnosis, treatment andfollow-up. Ann Oncol 2012;23 Suppl 7:vii11-9.

23. Molina R, Barak V, van Dalen A, et al. Tumor markers in breast cancer- European Group on Tumor Markers recommendations. Tumour Biol 2005;26(6):281-93.

24. Khatcheressian JL, Hurley P, Bantug E, et al. Breast cancer follow-up and management after primary treatment: American Society of Clinical Oncology clinical practice guideline update. J Clin Oncol 2013;31(7):961-5.

25. Ramsey SD, Henry NL, Gralow JR, et al. Tumor marker usage and medical care costs among older early-stage breast cancer survivors. J Clin Oncol 2015;33(2):149-55. 